\title{
ERDŐS' METHOD FOR DETERMINING THE IRRATIONALITY OF PRODUCTS
}

\author{
JAROSLAV HANČL ${ }^{\bowtie}$ and ONDŘEJ KOLOUCH
}

\author{
(Received 1 January 2011)
}

\begin{abstract}
This paper deals with a sufficient condition for the infinite product of rational numbers to be an irrational number. The condition requires only some conditions for convergence and does not use other properties like divisibility. The proof is based on an idea of Erdôs.
\end{abstract}

2010 Mathematics subject classification: primary 11J72.

Keywords and phrases: irrationality, infinite products.

\section{Introduction}

Following Erdős [4] we prove the following theorem.

THEOREM 1.1. Let $\left\{a_{n}\right\}_{n=1}^{\infty}$ be an increasing sequence of positive integers such that $\lim _{n \rightarrow \infty} a_{n}^{1 / 2^{n}}=\infty$. Then the number $\prod_{n=1}^{\infty}\left(1+\left(1 / a_{n}\right)\right)$ is irrational.

The authors do not know if the number $\prod_{n=1}^{\infty}\left(1+\left(1 /\left(2^{2^{n}}+1\right) a_{n}\right)\right)$ is irrational for all sequences $\left\{a_{n}\right\}_{n=1}^{\infty}$ of positive integers although we know from another theorem of Erdós [4] that the number $\sum_{n=1}^{\infty}\left(1 / 2^{2^{n}} a_{n}\right)$ is irrational for every sequence $\left\{a_{n}\right\}_{n=1}^{\infty}$ of positive integers. We are also not able to find a sequence $\left\{a_{n}\right\}_{n=1}^{\infty}$ of positive integers with $\lim _{n \rightarrow \infty} a_{n}^{1 / 2^{n}}=\infty$ and such that the number $\prod_{n=1}^{\infty}\left(1+\left(1 / a_{n}\right)\right)$ is algebraic. On the other side we know that $\prod_{n=1}^{\infty}\left(1+\left(1 / 2^{2^{n}}\right)\right)=4 / 3$. Erdôs [5] asked if the number $\sum_{n=1}^{\infty}\left(1 /\left(2^{2^{n}}+1\right) a_{n}\right)$ is irrational for all sequences $\left\{a_{n}\right\}_{n=1}^{\infty}$ of positive integers. Duverney [3] partially answered this question when he proved that if $\left\{a_{n}\right\}_{n=1}^{\infty}$ is a nondecreasing sequence of positive integers such that $\log a_{n}=o\left(2^{n}\right)$ then the number $\sum_{n=1}^{\infty}\left(1 /\left(2^{2^{n}}+1\right) a_{n}\right)$ is irrational. His result implies that the number $\sum_{n=1}^{\infty}\left(1 /\left(2^{2^{n}}+1\right)\right)$ is irrational. This can also be simply proved when we suppose that there exist positive integers $p$ and $q$ such that $\sum_{n=1}^{\infty}\left(1 /\left(2^{2^{n}}+1\right)\right)=p / q$, so the

The first author was supported by the grants no. ME09017 and MSM 6198898701.

(C) 2011 Australian Mathematical Publishing Association Inc. 0004-9727/2011 \$16.00 
number

$$
3 q \sum_{n=1}^{\infty} \frac{\prod_{n=1}^{N}\left(2^{2^{n}}+1\right)}{2^{2^{n}}+1}=3 q \sum_{n=1}^{N} \frac{\prod_{n=1}^{N}\left(2^{2^{n}}+1\right)}{2^{2^{n}}+1}+q+q 2^{-2^{N+2}}+o\left(2^{-2^{N+2}}\right)
$$

is an integer, which leads to a contradiction for a sufficiently large $N$.

Another partial solution was given by Badea [2] when he proved that if $\left\{a_{n}\right\}_{n=1}^{\infty}$ is a sequence of positive integers such that $a_{n+1}>2 a_{n}^{2}$ for all large $n$ then the number $\sum_{n=1}^{\infty}\left(1 /\left(2^{2^{n}}+1\right) a_{n}\right)$ is irrational.

There is a long history regarding the irrationality of infinite products. Badea [1] proved that if $\left\{a_{n}\right\}_{n=1}^{\infty}$ and $\left\{b_{n}\right\}_{n=1}^{\infty}$ are two sequences of positive integers such that

$$
a_{n+1}>\frac{b_{n+1}}{b_{n}} a_{n}^{2}+\frac{b_{n+1}\left(b_{n}-1\right)}{b_{n}} a_{n}+1-b_{n+1}
$$

holds for every sufficiently large $n$ then the number $\prod_{n=1}^{\infty}\left(1+\left(b_{n} / a_{n}\right)\right)$ is irrational. Using Brun's criterion, Laohakosol and Kuhapatanakul [12-14] worked in the spirit of Badea. Zhou and Lubinski [16] found some irrationality results regarding the numbers $\prod_{j=0}^{\infty}\left(1 \pm q^{-j} r+q^{-2 j} s\right)$. Zhou [15] proved the irrationality of certain multivariable infinite products. All this shows that the irrationality of infinite products is of substantial current interest.

Erdôs [4] proved that if $a=\left\{a_{n}\right\}_{n=1}^{\infty}$ is an increasing sequence of positive integers such that $\lim _{n \rightarrow \infty} a_{n}^{1 / 2^{n}}=\infty$ then the expressible set $E_{a}=\left\{\sum_{n=1}^{\infty}\left(1 / a_{n} c_{n}\right): c_{n} \in \mathbb{N}\right\}$ does not contain a rational number. Using this idea of Erdős, Hančl et al. [8] found some necessary conditions for the Lebesgue measure of $E_{a}$ to be equal to zero in the $p$-adic case. For other applications of this method see, for instance, $[6,7,9,10]$ or [11]. It seems that Erdős' idea still has great potential.

Theorem 2.1 is the main result. Its proof is quite involved but does not require any other knowledge beyond what has already been discussed. We denote the set of all positive integers by $\mathbb{Z}^{+}$.

\section{Main result}

THEOREM 2.1. Let $\varepsilon$ be a positive real number. Let $\left\{a_{n}\right\}_{n=1}^{\infty}$ and $\left\{b_{n}\right\}_{n=1}^{\infty}$ be two sequences of positive integers. Assume $\left\{a_{n}\right\}_{n=1}^{\infty}$ is nondecreasing and

$$
\limsup _{n \rightarrow \infty} a_{n}^{1 / 2^{n}}=\infty \text {. }
$$

Assume that for all sufficiently large $n$

$$
n^{1+\varepsilon} \leq a_{n}
$$

and

$$
b_{n} \leq a_{n}^{1 / \log ^{1+\varepsilon}} \log a_{n} .
$$

Then the number $x=\prod_{n=1}^{\infty}\left(1+\left(b_{n} / a_{n}\right)\right)$ is irrational. 


\section{Proofs}

Theorem 1.1 is an immediate consequence of Theorem 2.1.

Lemma 3.1. Let the sequence $\left\{a_{n}\right\}_{n=1}^{\infty}$ satisfy all conditions stated in Theorem 2.1. Then for every sufficiently large $n$

$$
\sum_{j=0}^{\infty} a_{n+j}^{1 /\left(\log ^{1+\varepsilon} \log a_{n+j}\right)-1}<a_{n}^{-\varepsilon / 2(1+\varepsilon)} .
$$

PROOF. From (2.2) and the fact that the sequence $\left\{a_{n}\right\}_{n=1}^{\infty}$ is nondecreasing we obtain

$$
\begin{aligned}
& \sum_{j=0}^{\infty} a_{n+j}^{1 /\left(\log ^{1+\varepsilon} \log a_{n+j}\right)-1} \\
& \left.=\sum_{n+j<a_{n}^{1 /(1+\epsilon)}} a_{n+j}^{1 /\left(\log ^{1+\varepsilon} \log a_{n+j}\right)-1}+\sum_{n+j \geq a_{n}^{1 /(1+\epsilon)}} a_{n+j}^{1 /(\log 1+\varepsilon} \log a_{n+j}\right)-1 \\
& \leq a_{n}^{1 /\left(\log ^{1+\varepsilon} \log a_{n}\right)-1} a_{n}^{1 /(1+\epsilon)}+\sum_{n+j \geq a_{n}^{1 /(1+\epsilon)}} a_{n+j}^{1 /\left(\log ^{1+\varepsilon} \log a_{n+j}\right)-1} \\
& \leq a_{n}^{1 /\left(\log ^{1+\varepsilon} \log a_{n}\right)-1} a_{n}^{1 /(1+\epsilon)}+\sum_{n+j \geq a_{n}^{1 /(1+\epsilon)}}(n+j)^{(1+\varepsilon)\left(1 /\left(\log ^{1+\varepsilon} \log (n+j)^{1+\varepsilon}\right)-1\right)} \\
& \leq a_{n}^{1 /\left(\log { }^{1+\varepsilon} \log a_{n}\right)-1} a_{n}^{1 /(1+\epsilon)}+\sum_{n+j \geq a_{n}^{1 /(1+\epsilon)}}(n+j)^{-(1+(2 \varepsilon / 3))} \leq a_{n}^{-\varepsilon / 2(1+\varepsilon)} .
\end{aligned}
$$

This concludes the proof.

Lemma 3.2. Let the sequence $\left\{a_{n}\right\}_{n=1}^{\infty}$ satisfy all conditions stated in Theorem 2.1 and instead of $(2.2)$ require

$$
2^{n}<a_{n}
$$

for every sufficiently large $n$. Then

$$
\left.\sum_{j=0}^{\infty} a_{n+j}^{1 /\left(\log ^{1+\varepsilon} \log a_{n+j}\right)-1}<a_{n}^{1 /(\log 1+(\varepsilon / 2)} \log a_{n}\right)-1
$$

holds for every sufficiently large $n$.

PROOF. From (3.2) and the fact that the sequence $\left\{a_{n}\right\}_{n=1}^{\infty}$ is nondecreasing we obtain

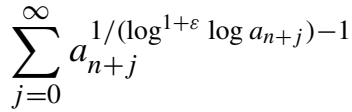

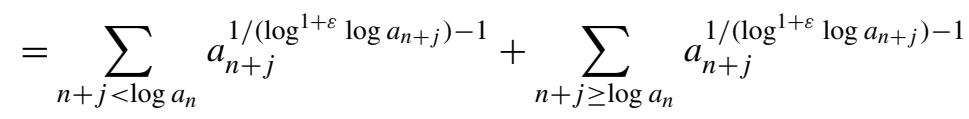

$$
\begin{aligned}
& \leq a_{n}^{1 /\left(\log ^{1+\varepsilon} \log a_{n}\right)-1} \log a_{n}+\sum_{n+j \geq \log a_{n}} a_{n+j}^{1 /\left(\log ^{1+\varepsilon} \log a_{n+j}\right)-1}
\end{aligned}
$$




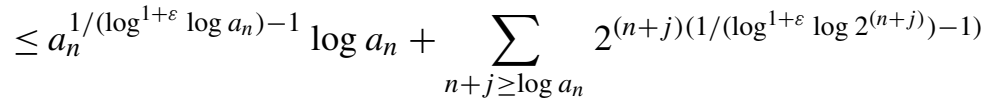

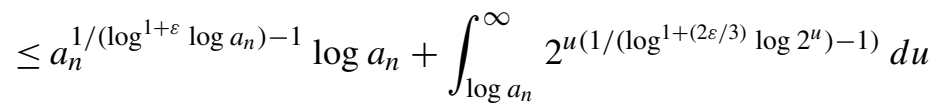

$$
\begin{aligned}
& \leq a_{n}^{1 /\left(\log ^{1+\varepsilon /(2)} \log a_{n}\right)-1} .
\end{aligned}
$$

This concludes the proof.

LEMMA 3.3. Let $\varepsilon^{*}$ and $\delta$ be two real numbers with $0 \leq \delta<1$ and $0<\varepsilon^{*}$. Let $\left\{a_{n}\right\}_{n=1}^{\infty}$ be a nondecreasing sequence of positive real numbers such that

$$
\limsup _{n \rightarrow \infty} a_{n}^{1 / n^{\delta} 2^{n}}=\infty
$$

Then for infinitely many $N$

$$
a_{N+1}^{1 /(N+1)^{\delta} 2^{N+1}}>\left(1+\frac{1}{N^{1+\left(\varepsilon^{*} / 4\right)}}\right) \max _{k=1, \ldots, N} a_{k}^{1 / k^{\delta} 2^{k}}
$$

and

$$
a_{N+1}>\left(1+\frac{1}{N^{1+\left(\varepsilon^{*} / 4\right)}}\right)^{(N+1)^{\delta} 2^{N+1}}\left(\prod_{n=1}^{N} a_{n}\right)\left(\prod_{n=1}^{N} a_{n}^{1 / n}\right)^{\delta / 2} .
$$

PROOF. From (3.4) we obtain that there exist infinitely many $N$ such that (3.5) holds. Otherwise there exists $N_{0}$ such that for each $N>N_{0}$

$$
\begin{aligned}
a_{N}^{1 / N^{\delta} 2^{N}} \leq & \left(1+\frac{1}{(N-1)^{1+\left(\varepsilon^{*} / 4\right)}}\right) \max _{k=1, \ldots, N-1} a_{k}^{1 / k^{\delta} 2^{k}} \\
\leq & \left(1+\frac{1}{(N-1)^{1+\left(\varepsilon^{*} / 4\right)}}\right)\left(1+\frac{1}{(N-2)^{1+\left(\varepsilon^{*} / 4\right)}}\right) \max _{k=1, \ldots, N-2} a_{k}^{1 / k^{\delta} 2^{k}} \\
\leq & \cdots \leq\left(1+\frac{1}{(N-1)^{1+\left(\varepsilon^{*} / 4\right)}}\right)\left(1+\frac{1}{(N-2)^{1+\left(\varepsilon^{*} / 4\right)}}\right) \cdots \\
& \times\left(1+\frac{1}{N_{0}^{1+\left(\varepsilon^{*} / 4\right)}}\right) \max _{k=1, \ldots, N_{0}-1} a_{k}^{1 / k^{\delta} 2^{k}} \leq \varepsilon^{*} \max _{k=1, \ldots, N_{0}-1} a_{k}^{1 / k^{\delta} 2^{k}}
\end{aligned}
$$

a contradiction with (3.4). From (3.5) we obtain that for infinitely many $N$

$$
\begin{aligned}
a_{N+1} & >\left(1+\frac{1}{N^{1+\left(\varepsilon^{*} / 4\right)}}\right)^{(N+1)^{\delta} 2^{N+1}}\left(\max _{k=1, \ldots, N} a_{k}^{1 / k^{\delta} 2^{k}}\right)^{(N+1)^{\delta} 2^{N+1}} \\
& >\left(1+\frac{1}{N^{1+\left(\varepsilon^{*} / 4\right)}}\right)^{(N+1)^{\delta} 2^{N+1}}\left(\max _{k=1, \ldots, N} a_{k}^{1 / k^{\delta} 2^{k}}\right)^{(N+1)^{\delta}\left(2^{N}+2^{N-1}+\cdots+2+1\right)} \\
& >\left(1+\frac{1}{N^{1+\left(\varepsilon^{*} / 4\right)}}\right)^{(N+1)^{\delta} 2^{N+1}}\left(\prod_{n=1}^{N} a_{n}\right)\left(\prod_{n=1}^{N} a_{n}^{1 / n}\right)^{\delta / 2} .
\end{aligned}
$$

This conlcudes the proof. 
Proof of Theorem 2.1. Assume that the number $x$ is a rational number. Then there exists $(p, q) \in \mathbb{Z}^{+} \times \mathbb{Z}^{+}$such that $x=p / q$. So for each $(P, Q) \in \mathbb{Z}^{+} \times \mathbb{Z}^{+}$ the number

$$
\left|q Q\left(x-\frac{P}{Q}\right)\right|=\left|q Q\left(\frac{p}{q}-\frac{P}{Q}\right)\right|=|p Q-P q|
$$

is an integer. To prove our theorem it is enough to find $(P, Q) \in \mathbb{Z}^{+} \times \mathbb{Z}^{+}$such that

$$
0<H=\left|q Q\left(x-\frac{P}{Q}\right)\right|<1 .
$$

Conditions (2.2) and (2.3) yield that the infinite product defining $x$ is convergent. Let $N$ be a sufficiently large positive integer. Set $Q_{N}=\prod_{n=1}^{N} a_{n}$ and

$$
P_{N}=\left(\prod_{n=1}^{N} a_{n}\right) \prod_{n=1}^{N}\left(1+\sum_{n=1}^{N} \frac{b_{n}}{a_{n}}\right) .
$$

Then

$$
\begin{aligned}
0<H_{N} & =\left|q Q_{N}\left(x-\frac{P_{N}}{Q_{N}}\right)\right| \\
& =\left|q\left(\prod_{n=1}^{N} a_{n}\right)\left(\prod_{n=1}^{\infty}\left(1+\frac{b_{n}}{a_{n}}\right)-\prod_{n=1}^{N}\left(1+\frac{b_{n}}{a_{n}}\right)\right)\right| \\
& \left.=q P_{N}\left(\prod_{n=N+1}^{\infty}\left(1+\frac{b_{n}}{a_{n}}\right)\right)-1\right) .
\end{aligned}
$$

From this and the fact that $x \geq P_{N} / Q_{N}$ we obtain that

$$
H_{N} \leq q Q_{N} x\left(\left(\prod_{n=N+1}^{\infty}\left(1+\frac{b_{n}}{a_{n}}\right)\right)-1\right) .
$$

This and the fact that the series $\sum_{n=1}^{\infty} b_{n} / a_{n}$ is absolutely convergent imply that there exists a positive real number $K$ which does not depend on $N$ and such that

$$
H_{N} \leq q Q_{N} x\left(\left(\prod_{n=N+1}^{\infty}\left(1+\frac{b_{n}}{a_{n}}\right)\right)-1\right) \leq K q Q_{N} x \sum_{n=N+1}^{\infty} \frac{b_{n}}{a_{n}} .
$$

From this, (2.3) and the definition of $Q_{N}$ we obtain that

$$
\left.H_{N} \leq K q Q_{N} x \sum_{n=N+1}^{\infty} \frac{b_{n}}{a_{n}} \leq K q x\left(\prod_{n=1}^{N} a_{n}\right) \sum_{n=N+1}^{\infty} a_{n}^{1 /(\log 1+\varepsilon} \log a_{n}\right)-1 .
$$

Now the proof falls into four cases. 
(1) Let us assume that (3.2) holds for every sufficiently large $n$ and there is a $\delta$ with $0<\delta<1$ and such that

$$
\limsup _{n \rightarrow \infty} a_{n}^{1 / n^{\delta} 2^{n}}=\infty
$$

This and Lemma 3.3 imply that there exist infinitely many $N$ such that

$$
a_{N+1}>\left(1+\frac{1}{N^{1+(\varepsilon / 4)}}\right)^{(N+1)^{\delta} 2^{N+1}}\left(\prod_{n=1}^{N} a_{n}\right)\left(\prod_{n=1}^{N} a_{n}^{1 / n}\right)^{\delta / 2} .
$$

From this, Lemma 3.2 and (3.8) we obtain that for infinitely many sufficiently large $N$

$$
\begin{aligned}
0 & \left.<H_{N}<K q x\left(\prod_{n=1}^{N} a_{n}\right) \sum_{n=N+1}^{\infty} a_{n}^{1 /(\log 1+\varepsilon} \log a_{n}\right)-1 \\
& \leq K q x\left(\prod_{n=1}^{N} a_{n}\right) a_{N+1}^{1 /\left(\log ^{1+(\varepsilon / 2)} \log a_{N+1}\right)-1} \\
& \leq K q x\left(\prod_{n=1}^{N} a_{n}\right) a_{N+1}^{1 /\left(\log ^{1+(\varepsilon / 2)} \log \left(\left(1+\left(1 / N^{1+(\varepsilon / 4)}\right)\right)^{(N+1)^{\delta} 2^{N+1}}\left(\prod_{n=1}^{N} a_{n}\right)\left(\prod_{n=1}^{N} a_{n}^{1 / n}\right)^{\delta / 2}\right)\right)-1} \\
& \leq\left(\prod_{n=1}^{N} a_{n}\right) a_{N+1}^{\left(1 / N^{1+(\varepsilon / 3)}\right)-1} \\
& \leq\left(\prod_{n=1}^{N} a_{n}\right)\left(\left(1+\frac{1}{N^{1+(\varepsilon / 4)}}\right)^{(N+1)^{\delta} 2^{N+1}}\left(\prod_{n=1}^{N} a_{n}\right)\left(\prod_{n=1}^{N} a_{n}^{1 / n}\right)^{\delta / 2}\right)^{\left(1 / N^{1+(\varepsilon / 3)}\right)-1} \\
& \leq\left(\prod_{n=1}^{N} a_{n}^{\left((1+(\delta / 2 n)) / N^{1+(\varepsilon / 3)}\right)-\delta / 2}\right)<1 .
\end{aligned}
$$

So (3.7) holds when we set $P=P_{N}, Q=Q_{N}$ and $H=H_{N}$.

(2) Let us assume that (3.2) holds for every sufficiently large $n$ and there is not a $\delta$ with $1>\delta>0$ and such that (3.9) holds. From this we see that for every $\delta>0$

$$
a_{n}<2^{n^{\delta} 2^{n}}
$$

holds for every sufficiently large $n$. Let $\delta$ be sufficiently small. Lemma 3.3 and (2.1) imply that for infinitely many $N$

$$
a_{N+1}>\left(1+\frac{1}{N^{1+(\varepsilon / 4)}}\right)^{2^{N+1}}\left(\prod_{n=1}^{N} a_{n}\right) .
$$

This, Lemma 3.2, (3.8) and (3.10) imply that for infinitely many $N$

$$
\begin{aligned}
0 & \left.<H_{N} \leq K q x\left(\prod_{n=1}^{N} a_{n}\right) \sum_{n=N+1}^{\infty} a_{n}^{1 /(\log 1+\varepsilon} \log a_{n}\right)-1 \\
& \leq K q x\left(\prod_{n=1}^{N} a_{n}\right) a_{N+1}^{1 /\left(\log ^{1+(\varepsilon / 2)} \log a_{N+1}\right)-1} \leq\left(\prod_{n=1}^{N} a_{n}\right) a_{N+1}^{\left(1 / N^{1+(\varepsilon / 3)}\right)-1}
\end{aligned}
$$




$$
\begin{aligned}
& \leq\left(\prod_{n=1}^{N} a_{n}\right)\left(\left(1+\frac{1}{N^{1+\left(\varepsilon^{*} / 4\right)}}\right)^{2^{N+1}}\left(\prod_{n=1}^{N} a_{n}\right)\right)^{\left(1 / N^{1+(\varepsilon / 3)}\right)-1} \\
& \leq\left(1+\frac{1}{N^{1+\left(\varepsilon^{*} / 4\right)}}\right)^{\left(\left(1 / N^{1+(\varepsilon / 3)}\right)-1\right) 2^{N+1}}\left(\prod_{n=1}^{N} a_{n}\right)^{1 / N^{1+(\varepsilon / 3)}} \\
& \leq\left(1+\frac{1}{N^{1+\left(\varepsilon^{*} / 4\right)}}\right)^{\left(\left(1 / N^{1+(\varepsilon / 3)}\right)-1\right) 2^{N+1}}\left(\prod_{n=1}^{N} 2^{n^{\delta} 2^{n}}\right)^{1 / N^{1+(\varepsilon / 3)}}<1 .
\end{aligned}
$$

So (3.7) holds when we set $P=P_{N}, Q=Q_{N}$ and $H=H_{N}$.

(3) Now let us assume that for infinitely many $n$

$$
a_{n} \leq 2^{n}
$$

and there is a $\delta$ with $0<\delta<1$ such that (3.9) holds. Let $A$ be a sufficiently large positive integer and $\delta$ sufficiently small. From (3.9) we see that there exists $n$ such that

$$
a_{n}^{1 / n^{\delta} 2^{n}}>A .
$$

Let $k$ be the least positive integer satisfying (3.12) and $s$ be the greatest positive integer less than $k$ such that (3.11) holds. Then

$$
a_{k}>A^{k^{\delta} 2^{k}}=2^{\left(\log _{2} A\right) k^{\delta} 2^{k}} .
$$

Hence there is a positive integer $n$ such that

$$
a_{n}^{1 / n^{\delta} 2^{n}} \geq 2
$$

Let $t$ be the least positive integer greater than $s$ such that (3.14) holds. It follows that for every $r=s, s+1, \ldots, t-1$

$$
a_{r}<2^{r^{\delta} 2^{r}}
$$

and

$$
a_{t} \geq 2^{t^{\delta} 2^{t}}
$$

Let us note that $k, s$ and $t$ depend on $A$ and if $A$ tends to infinity then also $k, s$ and $t$ tend to infinity. From (3.11), (3.15) and the fact that the sequence $\left\{a_{n}\right\}_{n=1}^{\infty}$ is nondecreasing we obtain that

$$
\begin{aligned}
\prod_{n=1}^{t-1} a_{n} & =\left(\prod_{n=1}^{s} a_{n}\right)\left(\prod_{n=s+1}^{t-1} a_{n}\right) \leq\left(\prod_{n=1}^{s} 2^{s}\right)\left(\prod_{n=s+1}^{t-1} 2^{n^{\delta} 2^{n}}\right) \\
& \leq 2^{s^{2}} 2^{t^{\delta} 2^{t}(1-(\delta / 2 t))-2^{s}} \leq 2^{t^{\delta} 2^{t}(1-(\delta / 2 t))} .
\end{aligned}
$$


Lemmas 3.1, 3.2 and (3.16) imply

$$
\begin{aligned}
& \left.\sum_{n=t}^{\infty} a_{n}^{1 /\left(\log ^{1+\varepsilon} \log a_{n}\right)-1}=\sum_{n=t}^{k-1} a_{n}^{1 /\left(\log ^{1+\varepsilon} \log a_{n}\right)-1}+\sum_{n=k}^{\infty} a_{n}^{1 /(\log 1+\varepsilon} \log a_{n}\right)-1 \\
& \leq a_{t}^{1 /\left(\log ^{1+(\varepsilon / 2)} \log a_{t}\right)-1}+a_{k}^{-\varepsilon / 2(1+\varepsilon)} \\
& \left.\left.\leq 2^{t^{\delta} 2^{t}(1 /(\log 1+(\varepsilon / 2)} \log 2^{t^{\delta} 2^{t}}\right)-1\right)+a_{k, 1}^{-\varepsilon / 2(1+\varepsilon)} \\
& \leq 2^{t^{\delta} 2^{t}\left(\left(1 / t^{1+(\varepsilon / 3)}\right)-1\right)}+a_{k, 1}^{-\varepsilon / 2(1+\varepsilon)} .
\end{aligned}
$$

From this, (3.8), (3.13) and (3.17) we obtain

$$
\begin{aligned}
0 & \left.<H_{t-1} \leq \operatorname{Kqx}\left(\prod_{n=1}^{t-1} a_{n}\right) \sum_{n=t}^{\infty} a_{n}^{1 /(\log 1+\varepsilon} \log a_{n}\right)-1 \\
& \leq K q x 2^{t^{\delta} 2^{t}(1-(\delta / 2 t))}\left(2^{t^{\delta} 2^{t}\left(\left(1 / t^{1+(\varepsilon / 3)}\right)-1\right)}+a_{k, 1}^{-\varepsilon / 2(1+\varepsilon)}\right) \\
& \leq K q x 2^{t^{\delta} 2^{t}(1-(\delta / 2 t))}\left(2^{t^{\delta} 2^{t}\left(\left(1 / t^{1+(\varepsilon / 3)}\right)-1\right)}+2^{-(\varepsilon / 2(1+\varepsilon))\left(\log _{2} A\right) k^{\delta} 2^{k}}\right)<1 .
\end{aligned}
$$

So (3.7) holds when we set $P=P_{t-1}, Q=Q_{t-1}$ and $H=H_{t-1}$.

(4) Finally let us assume that for infinitely many $n$ inequality (3.11) holds and there is no $\delta>0$ with $1>\delta>0$ and such that (3.9) holds. This implies that for every $\delta>0$ and sufficiently large $n$ inequality (3.10) holds. Let $\delta$ be sufficiently small and $A$ sufficiently large. From (2.1) we obtain

$$
a_{n}^{1 / 2^{n}}>A
$$

for infinitely many $n$. Let $k$ be the least positive integer satisfying (3.18). Then

$$
a_{k}>A^{2^{k}}=2^{\left(\log _{2} A\right) 2^{k}} .
$$

Let $s$ be the greatest positive integer less than $k$ such that (3.11) holds. From (2.1) and Lemma 3.3 we obtain that (3.5), with $\delta=0$, holds for infinitely many $N$. Let $t$ be the least positive integer greater than $s$ such that

$$
a_{t}^{1 / 2^{t}}>\left(1+\frac{1}{t^{1+(\varepsilon / 4)}}\right) \max _{j=s, \ldots, t-1} a_{j}^{1 / 2^{j}}
$$

and

$$
a_{r}^{1 / 2^{r}} \leq\left(1+\frac{1}{r^{1+(\varepsilon / 4)}}\right) \max _{j=s, \ldots, r-1} a_{j}^{1 / 2^{j}}
$$

for every $r=s+1, \ldots, t-1$. Inequality (3.20) and the fact that $a_{r} \leq 2^{s}$ for 
all $r=1,2, \ldots, s$ yield

$$
\begin{aligned}
a_{t} & >\left(\left(1+\frac{1}{t^{1+(\varepsilon / 4)}}\right) \max _{j=s, \ldots, t-1} a_{j}^{1 / 2^{j}}\right)^{2^{t}} \\
& =\left(1+\frac{1}{t^{1+(\varepsilon / 4)}}\right)^{2^{t}}\left(\max _{j=s, \ldots, t-1} a_{j}^{1 / 2^{j}}\right)^{2^{t}} \\
& \geq\left(1+\frac{1}{t^{1+(\varepsilon / 4)}}\right)^{2^{t}}\left(\max _{j=s, \ldots, t-1} a_{j}^{1 / 2^{j}}\right)^{2^{t-1}+2^{t-2}+\cdots+2^{s+1}} \\
& \geq\left(1+\frac{1}{t^{1+(\varepsilon / 4)}}\right)^{2^{t}}\left(\prod_{r=1}^{t-1} a_{r}\right) \\
& \geq\left(1+\frac{1}{t^{1+(\varepsilon / 4)}}\right)^{2^{t-1}}\left(\prod_{r=1}^{t-1} a_{r}\right) .
\end{aligned}
$$

From (3.21) we obtain

$$
\begin{aligned}
a_{r}^{1 / 2^{r}} & \leq\left(1+\frac{1}{r^{1+(\varepsilon / 4)}}\right) \max _{j=s, \ldots, r-1} a_{j}^{1 / 2^{j}} \\
& \leq\left(1+\frac{1}{r^{1+(\varepsilon / 4)}}\right)\left(1+\frac{1}{(r-1)^{1+(\varepsilon / 4)}}\right)_{j=s, \ldots, r-2} a_{j}^{1 / 2^{j}} \\
& \leq \cdots \leq \prod_{j=s+1}^{r}\left(1+\frac{1}{j^{1+(\varepsilon / 4)}}\right) a_{s}^{1 / 2^{s}} \leq D,
\end{aligned}
$$

where $1<D<2 \prod_{j=1}^{\infty}\left(1+\left(1 / j^{1+(\varepsilon / 4)}\right)\right)$ is a positive real constant which does not depend on $A$ and $k$. It follows that

$$
a_{r} \leq D^{2^{r}}=2^{\left(\log _{2} D\right) 2^{r}}
$$

for every $r=s+1, \ldots, t-1$. From this together with $a_{s}<2^{s}$ and the fact that the sequence $\left\{a_{n}\right\}_{n=1}^{\infty}$ is nondecreasing, we obtain

$$
\begin{aligned}
\prod_{r=1}^{t-1} a_{r} & =\left(\prod_{r=1}^{s} a_{r}\right)\left(\prod_{r=s+1}^{t-1} a_{r}\right) \leq\left(\prod_{r=1}^{s} 2^{s}\right)\left(\prod_{r=s+1}^{t-1} 2^{\left(\log _{2} D\right) 2^{r}}\right) \\
& \leq 2^{s^{2}} 2^{\left(\log _{2} D\right)\left(2^{t}-2^{s}\right)} \leq 2^{\left(\log _{2} D\right) 2^{t}} .
\end{aligned}
$$

Lemmas 3.1, 3.2, (3.22) and (3.24) imply

$$
\begin{aligned}
& \sum_{n=t}^{\infty} a_{n}^{1 /\left(\log ^{1+\varepsilon} \log a_{n}\right)-1}=\sum_{n=t}^{k-1} a_{n}^{1 /\left(\log ^{1+\varepsilon} \log a_{n}\right)-1}+\sum_{n=k}^{\infty} a_{n}^{1 /\left(\log ^{1+\varepsilon} \log a_{n}\right)-1} \\
& \quad \leq a_{t}^{1 /\left(\log ^{1+(\varepsilon / 2)} \log a_{t}\right)-1}+a_{k}^{-\varepsilon / 2(1+\varepsilon)}
\end{aligned}
$$




$$
\begin{aligned}
\leq & \left(\left(1+\frac{1}{t^{1+(\varepsilon / 4)}}\right)^{2^{t-1}}\left(\prod_{r=1}^{t-1} a_{r}\right)\right)^{1 /\left(\log ^{1+(\varepsilon / 2)} \log \left(\left(1+\left(1 / t^{1+(\varepsilon / 4)}\right)\right)^{2^{t-1}}\left(\prod_{r=1}^{t-1} a_{r}\right)\right)\right)-1} \\
& +a_{k}^{-\varepsilon / 2(1+\varepsilon)} \\
\leq & \left(\left(1+\frac{1}{t^{1+(\varepsilon / 4)}}\right)^{2^{t-1}}\left(\prod_{r=1}^{t-1} a_{r}\right)\right)^{\left(1 / t^{1+(\varepsilon / 3)}\right)-1}+a_{k}^{-\varepsilon / 2(1+\varepsilon)} \\
= & \left(1+\frac{1}{t^{1+(\varepsilon / 4)}}\right)^{2^{t-1}\left(\left(1 / t^{1+(\varepsilon / 3)}\right)-1\right)}\left(\prod_{r=1}^{t-1} a_{r}\right)^{1 / t^{1+(\varepsilon / 3)}}\left(\prod_{r=1}^{t-1} a_{r}\right)^{-1} \\
& +a_{k}^{-\varepsilon / 2(1+\varepsilon)} \\
\leq & \left(1+\frac{1}{t^{1+(\varepsilon / 4)}}\right)^{2^{t-1}\left(\left(1 / t^{1+(\varepsilon / 3)}\right)-1\right)} 2^{\left(\log _{2} D\right) 2^{t}\left(1 / t^{1+(\varepsilon / 3)}\right)}\left(\prod_{r=1}^{t-1} a_{r}\right)^{-1} \\
& +a_{k}^{-\varepsilon / 2(1+\varepsilon)} \\
\leq & 2^{-\left(1 / t^{1+(\varepsilon / 3)}\right) 2^{t}}\left(\prod_{r=1}^{t-1} a_{r}\right)^{-1}+a_{k}^{-\varepsilon / 2(1+\varepsilon)} .
\end{aligned}
$$

From this, (3.8), (3.19) and (3.24) we obtain

$$
\begin{aligned}
0<H_{t} & \leq K q x\left(\prod_{n=1}^{t-1} a_{n}\right) \sum_{n=t}^{\infty} a_{n}^{1 /\left(\log ^{1+\varepsilon} \log a_{n}\right)-1} \\
& \leq K q x\left(\prod_{n=1}^{t-1} a_{n}\right)\left(2^{-\left(1 / t^{1+(\varepsilon / 3)}\right) 2^{t}}\left(\prod_{r=1}^{t-1} a_{r}\right)^{-1}+a_{k}^{-\varepsilon / 2(1+\varepsilon)}\right) \\
& =\operatorname{Kqx}\left(2^{-\left(1 / t^{1+(\varepsilon / 3)}\right) 2^{t}}+\left(\prod_{n=1}^{t-1} a_{n}\right) a_{k}^{-\varepsilon / 2(1+\varepsilon)}\right) \\
& \leq K q x\left(2^{-\left(1 / t^{1+(\varepsilon / 3)}\right) 2^{t}}+2^{\left(\log _{2} D\right) 2^{t}} 2^{-(\varepsilon / 2(1+\varepsilon))\left(\log _{2} A\right) 2^{k}}\right)<1 .
\end{aligned}
$$

So (3.7) holds when we set $P=P_{t}, Q=Q_{t}$ and $H=H_{t}$.

\section{Acknowledgement}

The authors would like to thank James Carter of the College of Charleston for his help with their English.

\section{References}

[1] C. Badea, 'The irrationality of certain infinite products', Studia Univ. Babeş-Bolyai Math. 31(3) (1986), 3-8.

[2] C. Badea, 'The irrationality of certain infinite series', Glasg. Math. J. 29(2) (1987), 221-228. 
[3] D. Duverney, 'Sur les series de nombres rationnels a convergence rapide', C. R. Acad. Sci., Paris, Ser. I, Math. 328(7) (1999), 553-556.

[4] P. Erdôs, 'Some problems and results on the irrationality of the sum of infinite series', J. Math. Sci. 10 (1975), 1-7.

[5] P. Erdôs, 'Erdôs problem no. 6', in: 1995 Prague Midsummer Combinatorial Workshop, KAM Series (95-309) (ed. M. Klazar) (KAM MPP UK, Prague, 1995), p. 5.

[6] M. Genčev, 'Infinite product for $e^{6 \zeta(3)}$, Ann. Math. Sil. 21 (2007), 41-48, 2008.

[7] M. Genčev, 'Evaluation of infinite series involving special products and their algebraic characterization', Math. Slovaca 59(3) (2009), 365-378.

[8] J. Hančl, R. Nair, S. Sobková and J. Šustek, 'On expressible sets and p-adic numbers', Proc. Edinb. Math. Soc. (2), to appear.

[9] J. Hančl, P. Rucki and J. Šustek, 'A generalization of Sándor's theorem using iterated logarithms', Kumamoto J. Math. 19 (2006), 25-36.

[10] J. Hančl and S. Sobková, 'Special linearly unrelated sequences', J. Math. Kyoto Univ. 46(1) (2006), $31-45$.

[11] J. Hančl, J. Štěpnička and J. Šustek, 'Linearly unrelated sequences and Erdős' problem', Ramanujan J. 17(3) (2008), 331-342.

[12] V. Laohakosol and K. Kuhapatanakul, 'The reverse irrationality criteria of Brun and Badea', EastWest J. Math. Special vol. (2008), 217-234.

[13] V. Laohakosol and K. Kuhapatanakul, 'The irrationality criteria of Brun and Badea are essentially equivalent', in: Diophantine Analysis and Related Fields-DARF 2007/2008, AIP Conference Proceedings, 976 (American Institute of Physics, Melville, NY, 2008), pp. 144-159.

[14] V. Laohakosol and K. Kuhapatanakul, 'Irrationality criteria for infinite products', J. Comb. Number Theory 1(1) (2008), 49-57.

[15] P. Zhou, 'On the irrationality of a certain multivariable infinite product', Quaest. Math. 29(3) (2006), 351-365.

[16] P. Zhou and D. S. Lubinsky, 'On the irrationality of $\prod_{j=0}^{\infty}\left(1 \pm q^{-j} r+q^{-2 j} s\right)$ ', Analysis (Munich) 17(2-3) (1997), 129-153.

JAROSLAV HANČL, Department of Mathematics and Institute for Research and Applications of Fuzzy Modeling, University of Ostrava, 30. dubna 22,

70103 Ostrava 1, Czech Republic

e-mail: hancl@osu.cz

ONDŘEJ KOLOUCH, Department of Mathematics and Institute for Research and Applications of Fuzzy Modeling, University of Ostrava, 30. dubna 22,

70103 Ostrava 1, Czech Republic

e-mail: ondrej.kolouch@osu.cz 